\title{
An Enhanced Approach of CBIR using Gabor Wavelet and Edge Histogram Descriptor
}

\author{
Diksha Kurchaniya $^{1}$ and Punit K. Johari ${ }^{2}$ \\ 1, 2 Department of CSE/IT \\ Madhav Institute of Technology and Science \\ Gwalior, M.P., India \\ diksha5mits@gmail.com ${ }^{1}$,punitbhopal2006@gmail.com ${ }^{2}$
}

\begin{abstract}
In attention to the last decades with the rapid growth of digital images on the internet and digital image repository, retrieval of these images requires some efficient and effective image retrieval techniques accordance with the user perception. Many researchers have been proposed different image retrieval techniques. Content-Based Image Retrieval (CBIR) provides an effective approach to search and retrieve images from large databases, but to develop a CBIR system with an appropriate combination of low-level features is a big issue. To overcome these issues we used a combination of Gabor Wavelet and Edge Histogram Descriptor. The proposed approach is based on Gabor wavelet filter that have the capacity to collapse the filter reactions as per the scale and orientation of the textures. In Gabor wavelet, wavelets capture energy at a particular frequency and particular direction, we applied Gabor Wavelet filter in four different directions of the image such as-Horizontal, Vertical, Diagonal and Anti-Diagonal. Edges give the object illustration of an image and used as a feature descriptor for image retrieval. Spatial information about five different types of edges of the image is provided by EHD. EHD also gives geometrical information of image if same image has different colors. For calculating distance, Euclidean distance is used and average precision and recall are used for performance evaluation of proposed system. Experiment result shows that proposed method provides the improved precision over the existing method.
\end{abstract}

Keywords: Content Based Image Retrieval, Gabor wavelet filter, Edge Histogram Descriptor, Euclidean Distance

\section{Introduction}

In last decades, there are number of images captured by different sources, large number of digital images are used in different application areas such as- engineering, commerce, academics, hospital, crime prevention, government, architectures uses information in form of images. For fast and efficient retrieval of these image some image retrieval methods are used. The main goal of image retrieval is to find out the images from image database that is similar to query pattern given by the user on the basis of some similarity function. There are some types of image retrieval techniques: Text Based retrieval and Content Based retrieval. Text-based image retrieval (TBIR) is an image retrieval approach based on the manual annotation of images [14]. TBIR is a very fast and reliable system. But for large databases it takes several time to annotate every image another problem with TBIR is human perception means different people have different observation for same image. To overcome these limitations CBIR is another approach of image retrieval based on the visual feature such as color, shape and texture [1] [14]. These image features are known as low-level features. These visual features are stored in Multidimensional Feature vector

Received (May 6, 2017), Review Result (August 9, 2017), Accepted (August 21, 2017) 
and measuring the similarity between the feature vector of query image and database image feature vector.

\section{Existing Techniques}

1. Kato T [1] presented term an Image Retrieval approach in a conference organized by National Foundation of United States. He used color and shape as a visual feature of image for feature extraction of image retrieval system. Since then CBIR has been used to efficiently retrieve image by using its visual contents.

2. Chee Sun Won [2] firstly proposed a concept to retrieve the images from database on the basis of its shape, in which Edge Histogram Descriptors are used for image retrieval on the basis of shape of the image. The functionality of Edge Histogram descriptor is described in this paper.

3. M. Babu Rao [3] proposed an image retrieval framework that is based on dominant color feature and texture based Features for retrieving the images. Dominant color is used for color feature of an image and Motif co-occurrence matrix is used as a texture feature to extract visual content of image.

4. Sawet Somnugpong, kanokwan Khiewwan [5] proposed a new combination of low level feature for image retrieval. Color correlograms and edge direction histogram are used as a low level features. This combination gives spatial information of an image and prove that combination of low level features give better result than individual feature. Euclidean distance is used for similarity measurement.

5. Neelima Bagri and Punit k. Johari [6] proposed a paper on analysis of color and shape based better feature for analyzing the texture images. In this paper, several features of texture and shape are described on different databases and give the comparative study between them.

6. P. Hiremath and J. Pujari [8] proposed a system for image retrieval. Combination of texture, color and shape based features are used for image retrieval. They apply Color moments on Gabor filter that gives local description of texture, color and Shape information is captured by edges. Edges are computed using Gradient vector flow field then Invariant moments used.

7. R. Gali, M. deval, and R. Anand [9] have proposed texture, color and shape features extraction and combined these features to form feature vector of image and optimization is performed using GA and similarity measurement is used to perform retrieval from the database.

\section{Proposed Methodology}

Feature recognition and description are two essential components of different image retrieval applications. Feature extraction methods extract the visual features of the image such as- texture, color and shape. Multidimensional feature vector are used to store these features. In this proposed work, texture and shape based features extraction techniques are used [14]. 


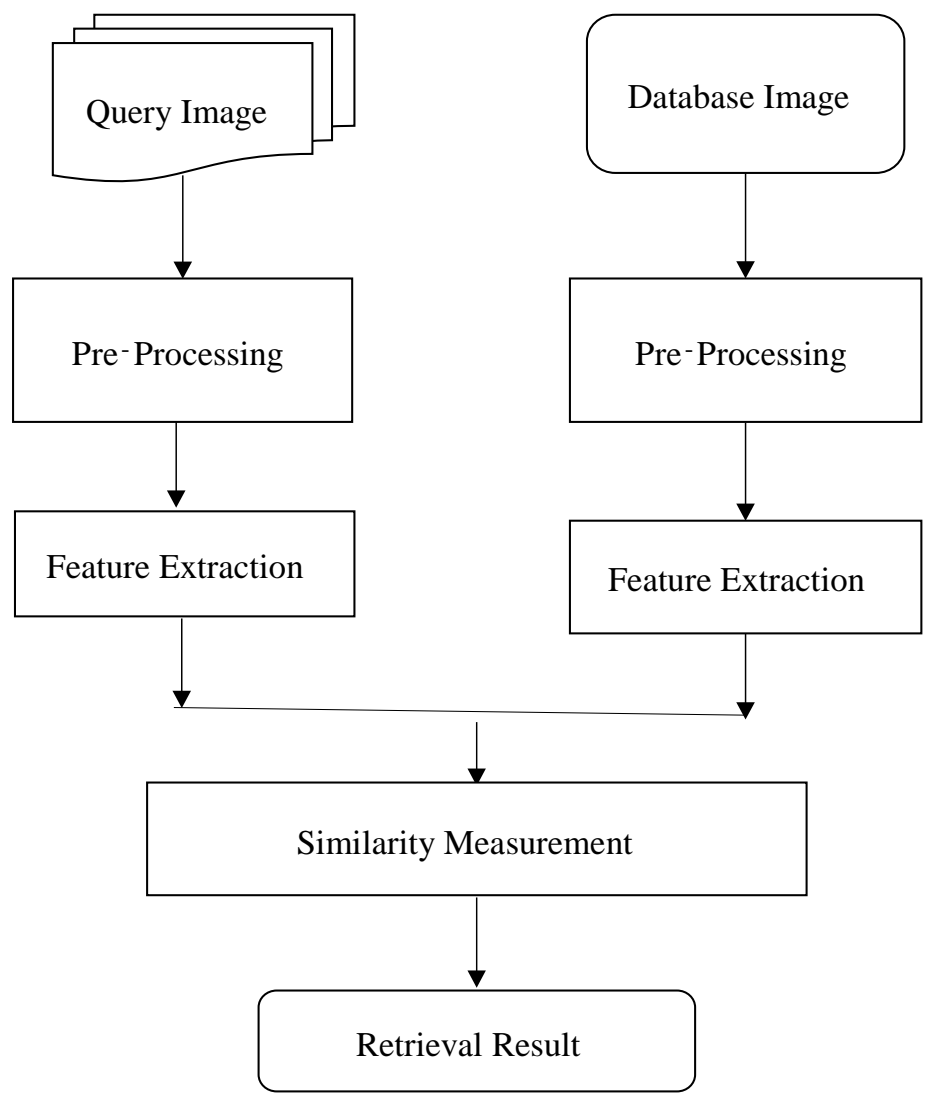

Figure 1. Image Retrieval System

\subsection{Gabor Wavelet Filter}

Gabor function is basically proposed in 1996 by Dennis Gabor to represent signals in the form of time and frequency domain. Gabor wavelet filter is an effective approach used for extraction of texture feature of an image [4]. Gabor Wavelet filter is a collection of wavelets, where each wavelet captures energy at a particular frequency and particular direction. It uses scale and orientation for line and bar detection. This scale and orientation property of Gabor filter is useful for texture feature analysis. The 2D Gabor filter to extract texture feature is as follows:

$$
g(x, y)=\frac{1}{2 \pi \sigma_{x} \sigma_{y}} \exp \left[-\frac{1}{2}\left(\frac{x^{2}}{\sigma_{x}^{2}}+\frac{y^{2}}{\sigma_{y}^{2}}\right)+2 \pi j W x\right]
$$

Where $\mathrm{W}$ is the frequency of sinusoid and $\sigma_{x}, \sigma_{y}$ are a standard deviation of Gaussian envelope.

Let assume $\mathrm{I}(x, y)$ be a grayscale distribution of image. For image $\mathrm{I}(x, y)$ its discrete Gabor wavelet transform using convolution is given below [8]:

$$
G_{m n}(x, y)=\sum_{s} \sum_{t} I(x-s, y-t) G_{m n}^{*}(s, t)
$$

Where s and t represents filter variables of mask size, $G_{m n}^{*}$ is the complex conjugate function $g_{m n}$ and $G_{m n}$ is the density value of Gabor kernel at orientation $\mathrm{m}$ and scale $\mathrm{n}$. Gabor filter is applied on given image $\mathrm{I}(x, y)$ for different orientation and scale then it calculate the array of magnitudes [18]: 


$$
(x, y)=\sum_{s} \sum_{t}\left|G_{m, n}(x, y)\right|
$$

Where $\mathrm{m}=0,1,2 \ldots \mathrm{M}-1$ and $\mathrm{n}=1,2 \ldots \mathrm{N}-1$.

These magnitudes are used to represent energy at different scale and orientation. Gabor filter is not rotation invariant it gives information about specific direction. To find the information of each direction we applied Gabor Wavelets on four different directions that are Horizontal, Vertical, Diagonal, and Anti-diagonal. Output filter computed using mean amplitude, standard deviation and wavelet moments.

\subsection{Edge Histogram Descriptor}

Edges are an essential component of image to represent the content of an image [2]. Edge Histogram Descriptor is a shape based feature used to extract edge information of image. MPEG defines edge histogram descriptor to retrieval of uniform edge descriptor and non-uniform texture descriptor. These descriptors describe edges in the form of a histogram of local edge descriptors. Image is distributed into $4 \times 4$ sub-images it is known as Local edge descriptor [5]. Then assign the level for four sub-blocks from 0 to 3 respectively and represent these gray levels at $(i, j)$ these image blocks are $a_{0}(i, j), a_{1}(i$, $j), a_{2}(i, j), a_{3}(i, j)$ respectively. It represent filter coefficient for vertical, horizontal, $45^{0}$ diagonal, and $135^{\circ}$ diagonal and non-directional edges [19]. Also, it can define filter coefficient for each direction as $\mathrm{f}_{\mathrm{v}}(k), \mathrm{f}_{h}(k), \mathrm{f}_{d-45}(k), \mathrm{f}_{d-135}(k), \mathrm{f}_{n}(k)$ [12].

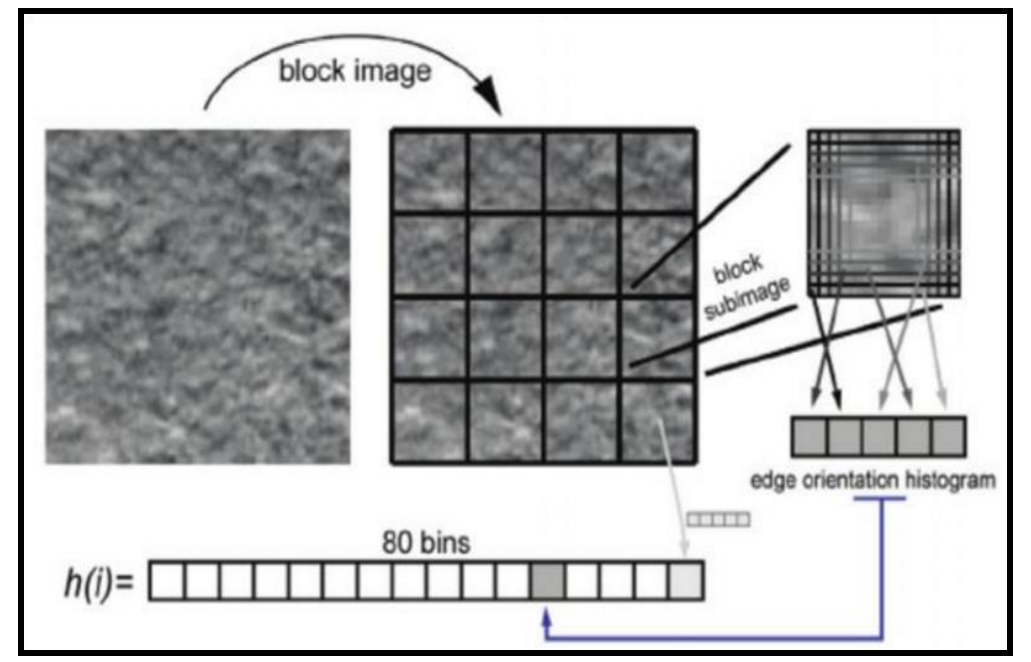

Figure 2. Define the Sub-Image and Image-Block

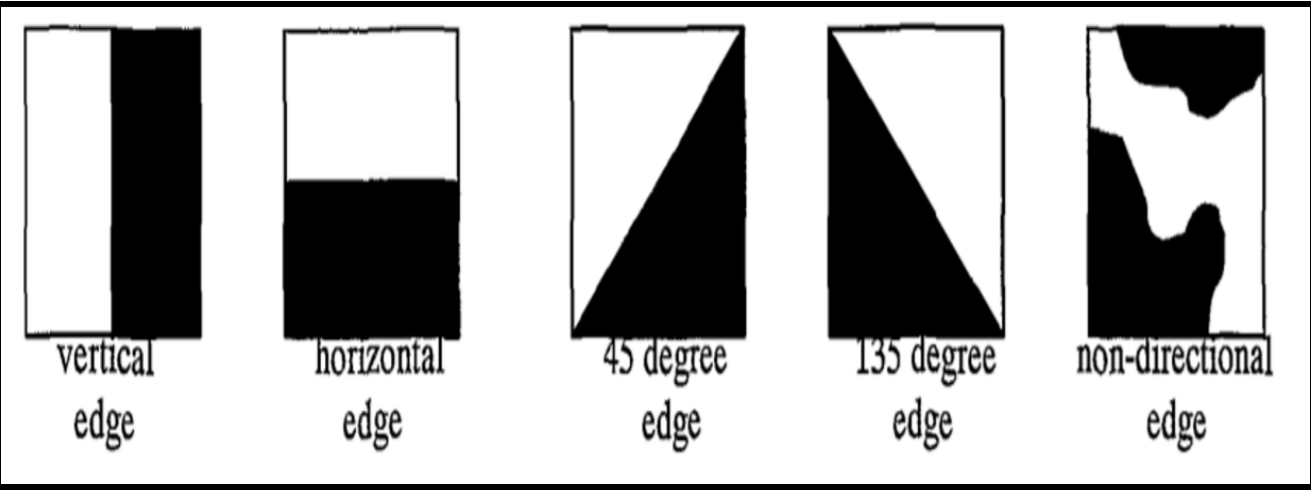

Figure 3. Five Types of Edges 
After extracting the edges, total number of bins are calculated in all edge types. For each sub-image is gives five histogram bins are known as Local edge histogram and five bins for image globally called as global edge histogram Now it has total [16x5=80 bins + $1 \times 5$ bins] 85 bins for edge histogram.

Now the respective magnitudes $\mathrm{m}_{\mathrm{v}}(i, j), \mathrm{m}_{h}(i, j), \mathrm{m}_{d-45}(i, j), \mathrm{m}_{d-135}(i, j), \mathrm{m}_{n}(i, j)$ or the $(i, j)^{\text {th }}$ image-block are as follows [13].

$$
\begin{aligned}
& m_{v}(\mathrm{i}, \mathrm{j})=\left\|\sum_{k=0}^{3} a_{k}(\mathrm{i}, \mathrm{j}) \times f_{v}(\mathrm{k})\right\| \\
& m_{h}(\mathrm{i}, \mathrm{j})=\left\|\sum_{k=0}^{3} a_{k}(\mathrm{i}, \mathrm{j}) \times f_{h}(\mathrm{k})\right\| \\
& m_{d-45}(\mathrm{i}, \mathrm{j})=\left\|\sum_{k=0}^{3} a_{k}(\mathrm{i}, \mathrm{j}) \times f_{d-45}(\mathrm{k})\right\| \\
& m_{d-135}(\mathrm{i}, \mathrm{j})=\left\|\sum_{k=0}^{3} a_{k}(\mathrm{i}, \mathrm{j}) \times f_{d-135}(\mathrm{k})\right\| \\
& m_{n}(\mathrm{i}, \mathrm{j})=\left\|\sum_{k=0}^{3} a_{k}(\mathrm{i}, \mathrm{j}) \times f_{n}(\mathrm{k})\right\|
\end{aligned}
$$

To calculate directional edge strength for each edge type, if the maximum value of all edge types is greater than the threshold value $\left(T_{\text {edge }}\right)$, then edge is considered otherwise no edge is considered [17].

$$
T_{\text {edge }}<\operatorname{Max}\left\{m_{v}(\mathrm{i}, \mathrm{j}), m_{h}(\mathrm{i}, \mathrm{j}), m_{d-45}(\mathrm{i}, \mathrm{j}), m_{d-135}(\mathrm{i}, \mathrm{j}), m_{n}(\mathrm{i}, \mathrm{j})\right\}
$$

\section{Proposed Algorithm}

Input: image for query and image database

Output: Number of images similar to query image

Step1: Input the RGB color image from the image database.

Step2: Initially resize all the image into $256 * 384$.

Step3: After Resizing all images convert them into RGB to Gray.

Step4: Extract the four different Direction from the image such as Horizontal $(\mathrm{H})$, Vertical (V), Diagonal (D) and Anti-Diagonal (AD).

Step5: Apply Gabor filter on first four edges and edge histogram descriptor on all four images.

Step6: Generate the feature vector Feature Vector of Gabor Wavelet and Feature Vector of Edge Histogram Descriptor.

Step7: Associate both feature vectors into a single feature vector.

Step8: To calculate the distance between feature vector of query image and feature vector database image Euclidean distance is used. 
Step9: After the calculating distance images are ranked from most relevant to irrelevant.

Step10: Retrieve top ranked images that are more similar to query image.

\section{Proposed Feature Extraction System}

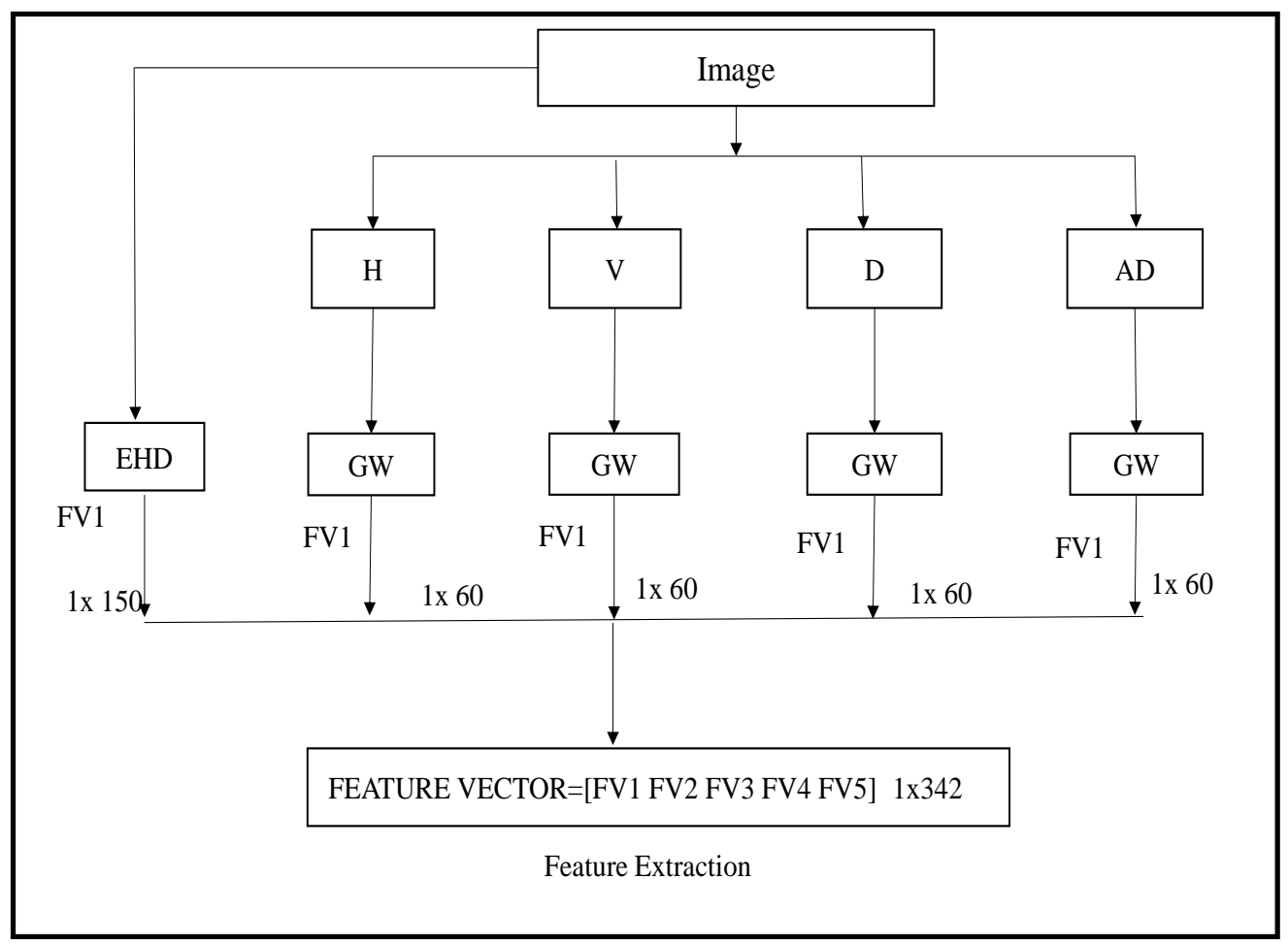

Figure 4. Proposed Feature Extraction System

\section{Experimental Result}

\subsection{Similarity Measurement}

Distance measure is an important task to calculate distance between two images and then rank these images from most relevant to irrelevant on the basis of distance. Retrieval performance of image retrieval system also depends on the similarity between feature vector of database image and feature vector of query image [20]. Euclidean distance is most extensively used for calculating the similarity between images. The following equation is used to calculate the similarity between the query image and database image [15] by using equation (9).

$$
D=\sqrt{\sum_{i=1}^{k}\left(q_{i}-d_{i}\right)^{2}}
$$

Where $\mathrm{q}_{\mathrm{i}}$ represents feature vector of the query image and $d_{i}$ represents feature vector of image database. $\mathrm{D}$ is the distance between the query image and database image and $\mathrm{k}$ represent the total number of element in each feature vector. 


\subsection{Experimental Database}

In proposed system, the database that we used in our evaluation is Wang database [16]. There are 1000 images with 10 categories in Wang database. We used 600 images of 6 categories. These are Africans, Beach, Architecture, Buses, Dinosaurs and Horse. Each category having 100 images approximately.

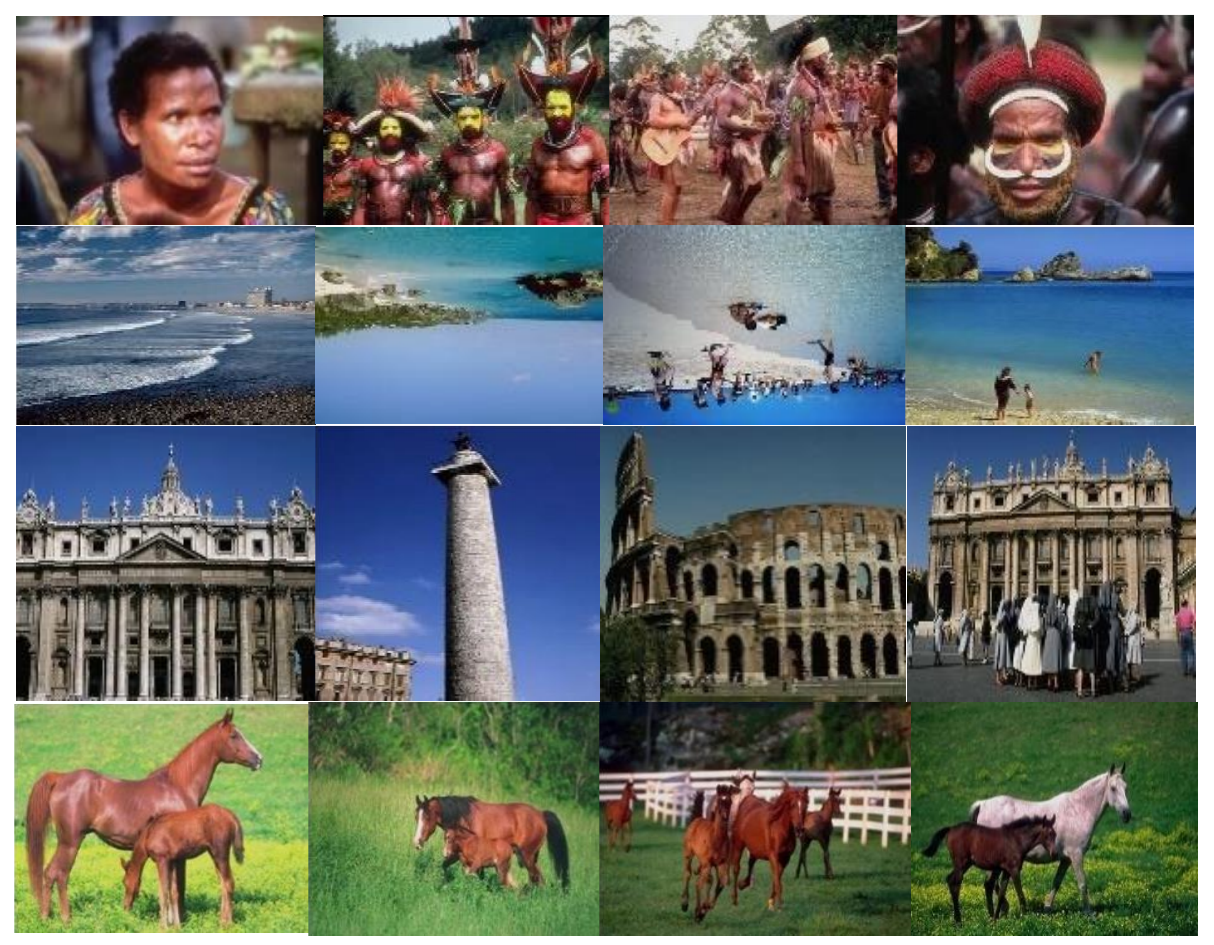

Figure 5. Four Image Semantic Types

\subsection{Performance Evaluation Matrices}

There are a large number of evaluation matrices are used to evaluate the retrieval performance [10]. Precision and Recall are used as an evaluation metric to evaluate the performance of the image retrieval system. Where precision and recall measure the availability of relevant images from the retrieved image in CBIR system.

$$
\begin{aligned}
& \text { Precision }=\frac{\text { Number of relevant images retrieved }}{\text { Total Number of images retrieved }} \\
& \text { Recall }=\frac{\text { Number of relevant images retrieved }}{\text { Total Number of images in database }}
\end{aligned}
$$

\subsection{Simulation and Results}

This proposed approach is implemented on MATLAB R2013b with PC core i3 $5^{\text {th }}$ generation CPU @ $2.00 \mathrm{GHz} 4$ GB of Ram capacity. MATLAB is high level simulation tool and have effective and interactive environment for programming, numeric computation and visualization. MATLAB tool is used for many applications like develop algorithms, examine the data, and create several models and applications. MATLAB have built in mathematical function which helps the programming easy. 
To check the efficiency of proposed system, it applied on some random images from different categories of WANG database. Images are chosen one by one as a query image to apply on this proposed system and top 5, 10, 20, 30 and 40 images are retrieved. Precision value is calculated for all retrieved images. Based on these precision values average precision is calculated. Proposed methodology gives better precision value in comparison of existing Methods.

Table 1. Average Precision for Proposed System

\begin{tabular}{|l|l|l|l|l|l|}
\hline \multirow{2}{*}{ Image } & \multicolumn{5}{|c|}{ Average Precision (\%) } \\
\cline { 2 - 6 } & $\mathrm{N}=5$ & $\mathrm{~N}=10$ & $\mathrm{~N}=20$ & $\mathrm{~N}=30$ & $\mathrm{~N}=40$ \\
\hline African & 0.94 & 0.92 & 0.83 & 0.78 & 0.73 \\
\hline Beach & 0.84 & 0.78 & 0.67 & 0.60 & 0.58 \\
\hline Building & 0.92 & 0.88 & 0.82 & 0.72 & 0.67 \\
\hline Bus & 1 & 0.98 & 0.95 & 0.92 & 0.88 \\
\hline Dinosaur & 1 & 1 & 1 & 1 & 0.99 \\
\hline Horse & 1 & 0.98 & 0.97 & 0.96 & 0.91 \\
\hline
\end{tabular}

Table 1 shows the result of Average Precision of top 5, 10, 20, 30 and 40 images retrieved using Proposed Methodology.

Table 2. Average Recall for Proposed System

\begin{tabular}{|l|l|l|l|l|l|}
\hline \multirow{2}{*}{ Image } & \multicolumn{5}{|c|}{ Average Recall (\%) } \\
\cline { 2 - 6 } & $\mathrm{N}=5$ & $\mathrm{~N}=10$ & $\mathrm{~N}=20$ & $\mathrm{~N}=30$ & $\mathrm{~N}=40$ \\
\hline African & 0.047 & 0.092 & 0.166 & 0.234 & 0.29 \\
\hline Beach & 0.042 & 0.078 & 0.134 & 0.18 & 0.21 \\
\hline Building & 0.046 & 0.088 & 0.164 & 0.216 & 0.27 \\
\hline Bus & 0.05 & 0.098 & 0.19 & 0.276 & 0.35 \\
\hline Dinosaur & 0.05 & 0.05 & 0.05 & 0.05 & 0.39 \\
\hline Horse & 0.05 & 0.098 & 0.194 & 0.288 & 0.36 \\
\hline
\end{tabular}

Table 2 shows the result of the average recall of top 5, 10, 20, 30 and 40 images using Proposed Methodology.

Table 3. Comparison with Proposed by Other's

\begin{tabular}{|c|c|c|c|c|}
\hline \multirow{2}{*}{ Category } & Method [3] & Method [5] & Method [12] & $\begin{array}{c}\text { Proposed } \\
\text { Method }\end{array}$ \\
\cline { 2 - 5 } & $\begin{array}{c}\text { Average } \\
\text { Precision }\end{array}$ & $\begin{array}{c}\text { Average } \\
\text { Precision }\end{array}$ & $\begin{array}{c}\text { Average } \\
\text { Precision }\end{array}$ & $\begin{array}{c}\text { Average } \\
\text { Precision }\end{array}$ \\
\hline Africans & 0.56 & 0.67 & 0.59 & $\mathbf{0 . 8 4}$ \\
\hline Beach & 0.53 & 0.59 & 0.75 & $\mathbf{0 . 6 8}$ \\
\hline Building & 0.61 & 0.58 & 0.73 & $\mathbf{0 . 8 0}$ \\
\hline Buses & 0.89 & 0.94 & 0.96 & $\mathbf{0 . 9 5}$ \\
\hline Dinosaur & 0.98 & 0.99 & 0.97 & $\mathbf{0 . 9 9}$ \\
\hline Horses & 0.78 & 0.93 & 0.69 & $\mathbf{0 . 9 6}$ \\
\hline $\begin{array}{c}\text { Overall } \\
\text { Average }\end{array}$ & 0.725 & 0.783 & 0.781 & $\mathbf{0 . 8 7}$ \\
\hline
\end{tabular}


Table 3 shows the comparison between Method using in [3], [5], [12] and Proposed Methodology.

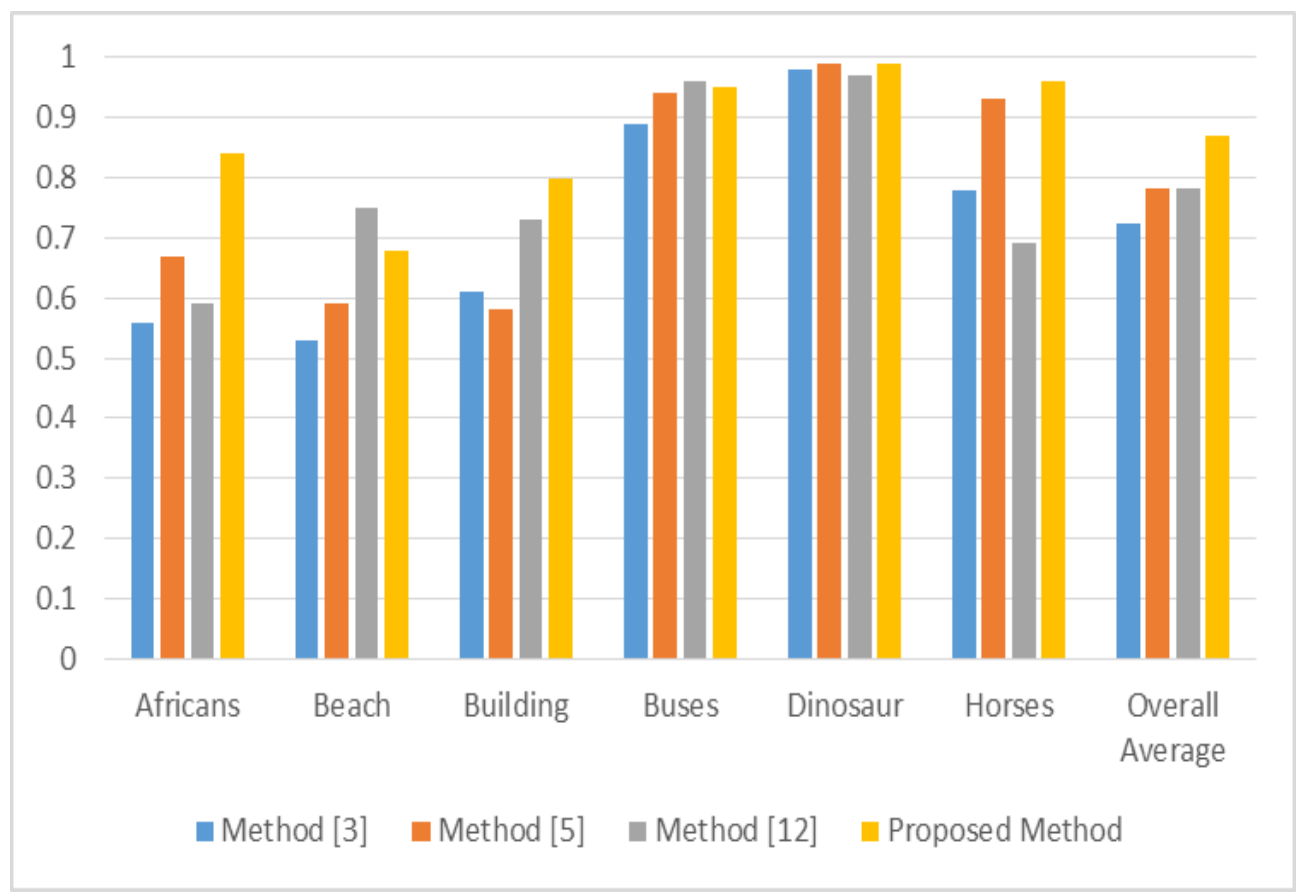

Figure 6. Comparison Graph of Average Precision

In Figure 6, the comparative graph showed comparison between Method used in [3], [5], [12] and Proposed System.

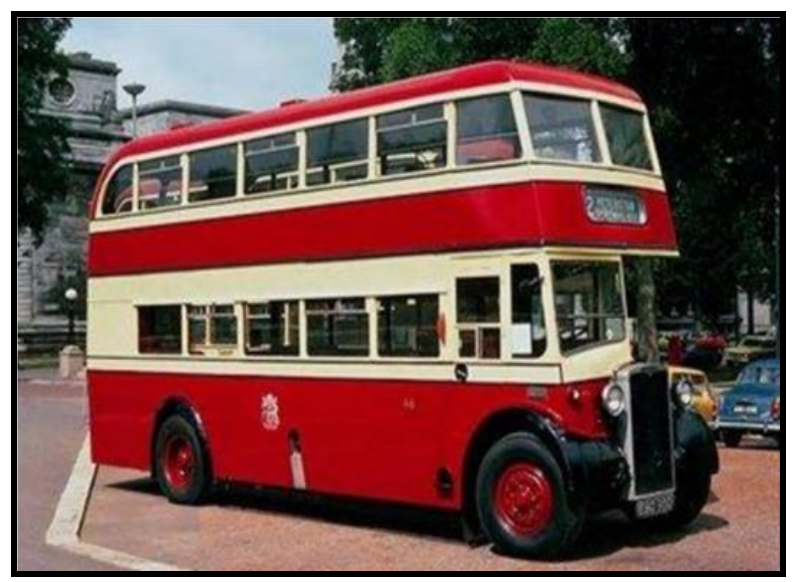

Figure 7. Query Image 


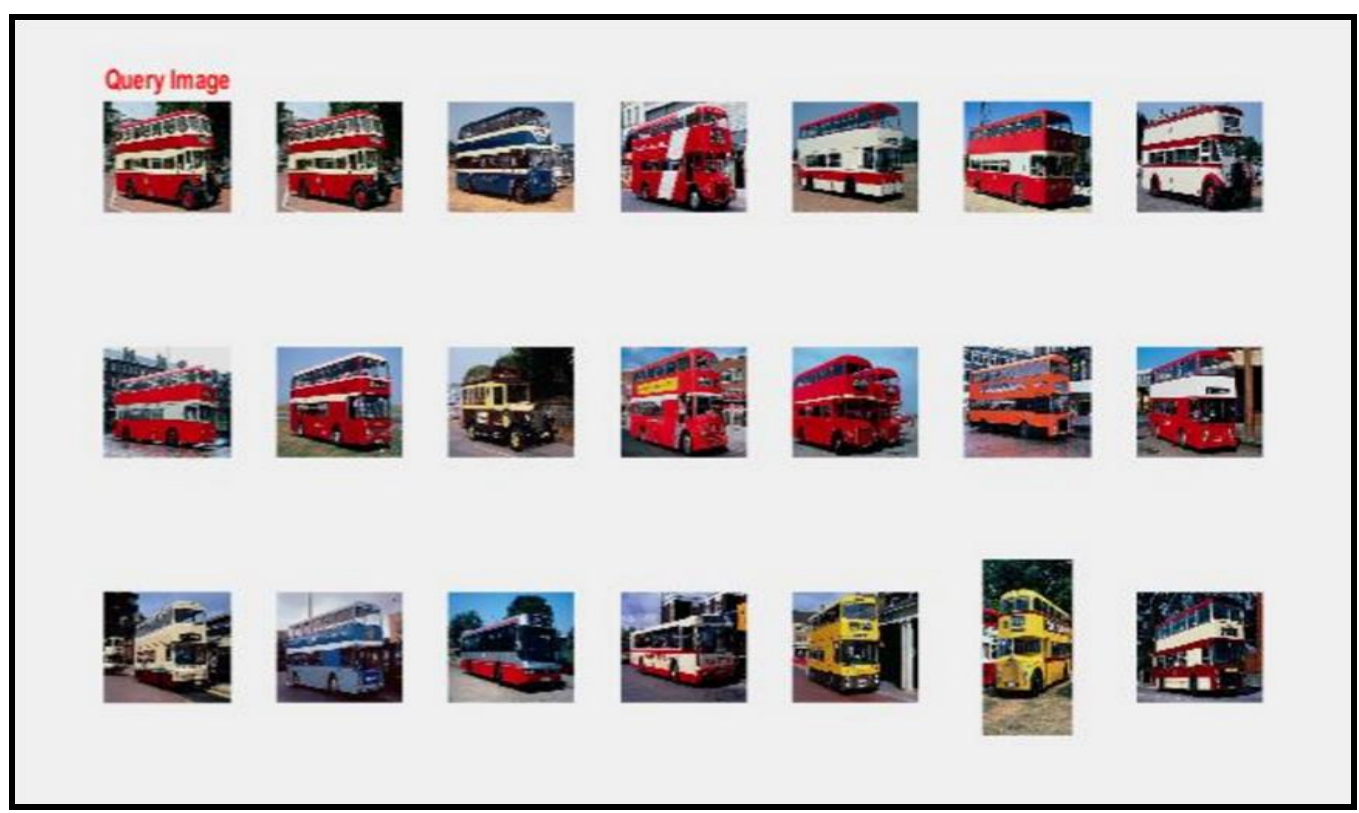

Figure 8. Retrieved Result for Bus Image Based on Proposed Method

Figure 8 shows first 20 retrieved image for the given query image of bus using proposed methodology.

\section{Conclusion and Future Scope}

In this proposed system a combination of two compatible low-level features is described. This proposed system is basically focused on simply texture and shape-based feature to show the efficiency of texture and shape based method over the color. Based on the analysis of Gabor filter we applied Gabor Wavelets on four directions that are Horizontal, Vertical, Diagonal, and Anti-diagonal. We used Edge Histogram Descriptor for shape based feature. Gabor Wavelet filter and EHD is used to find the better efficiency and improved retrieval precision. In future, to increase the efficiency of proposed retrieval system further we can use relevance feedback and some optimization techniques.

\section{References}

[1] T. Kato, "Database architecture for content-based image retrieval", in Image Storage and Retrieval Systems, Proc SPIE 1662, (1992) pp112-123.

[2] C. S.Won, D. K. Park and Y. S. Jeon, "an efficient use of MPEG-7 Color Layout and Edge Histogram Descriptors", proceeding of the ACM workshop on multimedia, (2000), pp. 51-54.

[3] M. Babu Rao, D. Prabhakar Rao, A. Govardhan, "Content-based Image Retrieval System based on Dominant Color and Texture Features", International Journal of Computer Applications, ISSN: 0975 8887, vol. 18, no.6, (2014).

[4] D. Gabor, "Theory of communication. Journal of the Institution of Electrical Engineers", - Part III: Radio and Communication Engineering, vol. 93, no. 26, (1946), pp. 429-457.

[5] S. Somnugpong and K. Khiewwan, "Content Based Image Retrieval using a Combination of Color Correlograms and Edge Direction Histogram", $13^{\text {th }}$ International Joint Conference on Computer Science and Software Engineering,DOI: 10.1109, IEEE, (2016).

[6] N. Bagri and P. K. Johari, "A Comparative Study on Feature Extraction using Texture and Shape for Content Based Image Retrieval", International Journal of Advanced Science and Technology, ISSN: 2005-4238, vol.80, (2015) pp.41-52.

[7] S. Jayaraman, S. Esakkirajan and T. Veerakumar, "Digital image processing", Tata McGraw hill education private limited, ISBN (13): 978-0-07-014479-8.

[8] P. Hiremath and J. Pujari, "Content Based Image Retrieval using Color Texture and Shape features", $15^{\text {th }}$ international conference on advanced computing and communication, (2007).

[9] R. Gali, M. deval and R. Anand, "Genetic Algorithm for Content-Based Image Retrieval", Forth Conference on Computational Intelligence, Communication System and Network, (2012) 
[10] R. Jain and P. K. Johari, "An Improved Approach of CBIR using Color Based HSV Quantization and Shape Based Edge Detection Algorithm", IEEE International Conference on Recent Trends in Electronics Information Communication Technology, ISBN: 978-1-5090-0774-5, DOI 10.1109, (2016), pp.1970-1995.

[11] D. Kurchaniya and P.K. Johari, "An Efficient Approach for Image Retrieval using Particle Swarm Optimization", International Journal of Computer Science and Engineering, e-ISSN: 2343-2693, vol. 5, Issue 6, (2017).

[12] M. D. Chaudhary and A.B. Upadhyay, "Integrating Shape and Edge Histogram Descriptor with Stationary Wavelet Transform for Effective Content Based Image Retrieval", International Conference on Circuit, Power and Computing Technologies [ICCPCT] ISBN: 978-14799-2397-7, (2014).

[13] P. Mane and N. G. Bawane, "Comparative Performance Evaluation of Edge Histogram Descriptors and Color Structure Descriptors in Content-based Image Retrieval", National Conference on Innovative Paradigms in Engineering \& Technology, (2013).

[14] F. Long and H. Zhang, "Fundamentals of Content-Based Image Retrieval".

[15] R. Devesh, J. Jha and R. Jayaswal, "Retrieval of Monument Images through ACO Optimization Approach”, International Research Journal of Engineering and Technology, vol. 4 Issue 7, (2017).

[16] B. Wang, X. Zhang and N. Li, "Relevance Feedback Technique For Content Based Image Retrieval Using Neural Network Learning", Proceeding of the Fifth International Conference on Machine Learning and Cybernetics, Dalian, (2006).

[17] R. Jayaswal, J. Jha and R. Devesh, "An Effective Method of Image Mining using k-Medoid Clustering Technique", International Journal of Computer Science and Engineering, e-ISSN: 2343-2693, vol. 5, Issue 6, (2017).

[18] A. Singla1 and M. Garg, "CBIR Approach Based On Combined HSV, Auto Correlogram, Color Moments and Gabor Wavelet", International Journal of Engineering and Computer Science ISSN: 23197242, Page No. 9007-9012, vol.3, Issue 10, (2014).

[19] M. A. Ansari and M. Dixit, "An Image Retrieval Framework: A Review", International Journal of Advanced Research in Computer Science, June, ISSN: 0976-5697, vol. 8, no. 4, (2017)

[20] D. Kurchaniya and P. K. Johary, "Analysis of Different Similarity Measures in Image Retrieval Based on Texture and Shape", International Research Journal of Engineering and Technology (IRJET), eISSN: 2395 -0056, p-ISSN: 2395-0072, vol. 04 Issue: 04, (2017). 
International Journal of Signal Processing, Image Processing and Pattern Recognition Vol. 10, No. 10 (2017) 